\title{
On the Ulam stability of mixed type QA mappings in IFN-spaces
}

\author{
Abdulrahman S Al-Fhaid and Syed Abdul Mohiuddine*
}

"Correspondence:

mohiuddine@gmail.com

Department of Mathematics,

Faculty of Science, King Abdulaziz

University, P.O. Box 80203, Jeddah,

21589, Saudi Arabia

\begin{abstract}
We give Ulam-type stability results concerning the quadratic-additive functional equation in intuitionistic fuzzy normed spaces.
\end{abstract}

Keywords: $t$-norm; $t$-conorm; quadratic-additive functional equation; intuitionistic fuzzy normed space; Hyers-Ulam stability

\section{Introduction}

In 1940, Ulam [1] proposed the following stability problem: 'When is it true that a function which satisfies some functional equation approximately must be close to one satisfying the equation exactly?? Hyers [2] gave the first affirmative partial answer to the question of Ulam for Banach spaces. Aoki [3] presented a generalization of Hyers results by considering additive mappings, and later on Rassias [4] did for linear mappings by considering an unbounded Cauchy difference. The paper of Rassias has significantly influenced the development of what we now call the Hyers-Ulam-Rassias stability of functional equations. Various extensions, generalizations and applications of the stability problems have been given by several authors so far; see, for example, [5-24] and references therein.

The notion of intuitionistic fuzzy set introduced by Atanassov [25] has been used extensively in many areas of mathematics and sciences. Using the idea of intuitionistic fuzzy set, Saadati and Park [26] presented the notion of intuitionistic fuzzy normed space which is a generalization of the concept of a fuzzy metric space due to Bag and Samanta [27]. The authors of [28-34] defined and studied some summability problems in the setting of an intuitionistic fuzzy normed space.

In the recent past, several Hyers-Ulam stability results concerning the various functional equations were determined in [35-46], respectively, in the fuzzy and intuitionistic fuzzy normed spaces. Quite recently, Alotaibi and Mohiuddine [47] established the stability of a cubic functional equation in random 2-normed spaces, while the notion of random 2normed spaces was introduced by Goleț [48] and further studied in [49-51].

The Hyers-Ulam stability problems of quadratic-additive functional equation

$$
f(x+y+z)+f(x)+f(y)+f(z)=f(x+y)+f(y+z)+f(x+z)
$$

under the approximately even (or odd) condition were established by Jung [52] and the solution of the above functional equation where the range is a field of characteristic 0 was determined by Kannappan [53]. In this paper we determine the stability results concerning 
the above functional equation in the setting of intuitionistic fuzzy normed spaces. This work indeed presents a relationship between two various disciplines: the theory of fuzzy spaces and the theory of functional equations.

\section{Definitions and preliminaries}

We shall assume throughout this paper that the symbol $\mathbb{N}$ denotes the set of all natural numbers.

A binary operation $*:[0,1] \times[0,1] \rightarrow[0,1]$ is said to be a continuous $t$-norm if it satisfies the following conditions:

(a) $*$ is associative and commutative, (b) $*$ is continuous, (c) $a * 1=a$ for all $a \in[0,1]$, (d) $a * b \leq c * d$ whenever $a \leq c$ and $b \leq d$ for each $a, b, c, d \in[0,1]$.

A binary operation $\diamond:[0,1] \times[0,1] \rightarrow[0,1]$ is said to be a continuous $t$-conorm if it satisfies the following conditions:

$\left(\mathrm{a}^{\prime}\right) \diamond$ is associative and commutative, $\left(\mathrm{b}^{\prime}\right) \diamond$ is continuous, $\left(\mathrm{c}^{\prime}\right) a \diamond 0=a$ for all $a \in[0,1]$, $\left(\mathrm{d}^{\prime}\right) a \diamond b \leq c \diamond d$ whenever $a \leq c$ and $b \leq d$ for each $a, b, c, d \in[0,1]$.

The five-tuple $(X, \mu, \nu, *, \diamond)$ is said to be intuitionistic fuzzy normed spaces (for short, IFN-spaces) [26] if $X$ is a vector space, $*$ is a continuous $t$-norm, $\diamond$ is a continuous $t$ conorm, and $\mu, v$ are fuzzy sets on $X \times(0, \infty)$ satisfying the following conditions. For every $x, y \in X$ and $s, t>0$,

(i) $\mu(x, t)+v(x, t) \leq 1$,

(ii) $\mu(x, t)>0$,

(iii) $\mu(x, t)=1$ if and only if $x=0$,

(iv) $\mu(\alpha x, t)=\mu\left(x, \frac{t}{|\alpha|}\right)$ for each $\alpha \neq 0$,

(v) $\mu(x, t) * \mu(y, s) \leq \mu(x+y, t+s)$,

(vi) $\mu(x, \cdot):(0, \infty) \rightarrow[0,1]$ is continuous,

(vii) $\lim _{t \rightarrow \infty} \mu(x, t)=1$ and $\lim _{t \rightarrow 0} \mu(x, t)=0$,

(viii) $v(x, t)<1$,

(ix) $v(x, t)=0$ if and only if $x=0$,

(x) $v(\alpha x, t)=v\left(x, \frac{t}{|\alpha|}\right)$ for each $\alpha \neq 0$,

(xi) $v(x, t) \diamond v(y, s) \geq v(x+y, t+s)$,

(xii) $v(x, \cdot):(0, \infty) \rightarrow[0,1]$ is continuous,

(xiii) $\lim _{t \rightarrow \infty} v(x, t)=0$ and $\lim _{t \rightarrow 0} v(x, t)=1$.

In this case $(\mu, \nu)$ is called an intuitionistic fuzzy norm. For simplicity in notation, we denote the intuitionistic fuzzy normed spaces by $(X, \mu, \nu)$ instead of $(X, \mu, \nu, *, \diamond)$. For example, let $(X,\|\cdot\|)$ be a normed space, and let $a * b=a b$ and $a \diamond b=\min \{a+b, 1\}$ for all $a, b \in[0,1]$. For all $x \in X$ and every $t>0$, consider

$$
\mu(x, t):=\frac{t}{t+\|x\|} \quad \text { and } \quad v(x, t):=\frac{\|x\|}{t+\|x\|} .
$$

Then $(X, \mu, v)$ is an intuitionistic fuzzy normed space.

The notions of convergence and Cauchy sequence in the setting of IFN-spaces were introduced by Saadati and Park [26] and further studied by Mursaleen and Mohiuddine [30].

Let $(X, \mu, v)$ be an intuitionistic fuzzy normed space. Then the sequence $x=\left(x_{k}\right)$ is said to be: 
(i) Convergent to $L \in X$ with respect to the intuitionistic fuzzy norm $(\mu, v)$ if, for every $\epsilon>0$ and $t>0$, there exists $k_{0} \in \mathbb{N}$ such that $\mu\left(x_{k}-L, t\right)>1-\epsilon$ and $\nu\left(x_{k}-L, t\right)<\epsilon$ for all $k \geq k_{0}$. In this case, we write $(\mu, v)-\lim x_{k}=L$ or $x_{k} \stackrel{(\mu, v)}{\longrightarrow} L$ as $k \rightarrow \infty$.

(ii) Cauchy sequence with respect to the intuitionistic fuzzy norm $(\mu, v)$ if, for every $\epsilon>0$ and $t>0$, there exists $k_{0} \in \mathbb{N}$ such that $\mu\left(x_{k}-x_{\ell}, t\right)>1-\epsilon$ and $\nu\left(x_{k}-x_{\ell}, t\right)<\epsilon$ for all $k, \ell \geq k_{0}$. An IFN-space $(X, \mu, \nu)$ is said to be complete if every Cauchy sequence in $(X, \mu, v)$ is convergent in the IFN-space. In this case, $(X, \mu, v)$ is called an intuitionistic fuzzy Banach space.

\section{Stability of a quadratic-additive functional equation in the IFN-space}

We shall assume the following abbreviation throughout this paper:

$$
D f(x, y, z)=f(x+y+z)-f(x+y)-f(y+z)-f(x+z)+f(x)+f(y)+f(z) .
$$

Theorem 3.1 Let $X$ be a linear space and $(X, \mu, v)$ be an IFN-space. Suppose that $f$ is an intuitionistic fuzzy q-almost quadratic-additive mapping from $(X, \mu, v)$ to an intuitionistic fuzzy Banach space $\left(Y, \mu^{\prime}, v^{\prime}\right)$ such that

$$
\left.\begin{array}{l}
\mu^{\prime}(D f(x, y, z), s+t+u) \geq \mu\left(x, s^{q}\right) * \mu\left(y, t^{q}\right) * \mu\left(z, u^{q}\right) \quad \text { and } \\
v^{\prime}(D f(x, y, z), s+t+u) \leq v\left(x, s^{q}\right) \diamond v\left(y, t^{q}\right) \diamond v\left(z, u^{q}\right)
\end{array}\right\}
$$

for all $x, y, z \in X$ and $s, t, u>0$, where $q$ is a positive real number with $q \neq \frac{1}{2}, 1$. Then there exists a unique quadratic-additive mapping $T: X \rightarrow Y$ such that

$$
\begin{aligned}
& \mu^{\prime}(T(x)-f(x), t) \geq \begin{cases}\sup _{t^{\prime}<t} \mu\left(x,\left(\frac{2-2^{p}}{3}\right)^{q} t^{\prime q}\right) & \text { if } q>1, \\
\sup _{t^{\prime}<t} \mu\left(x,\left(\frac{\left(4-2^{p}\right)\left(2-2^{p}\right)}{6}\right)^{q} t^{\prime q}\right) & \text { if } \frac{1}{2}<q<1, \\
\sup _{t^{\prime}<t} \mu\left(x,\left(\frac{2^{p}-4}{3}\right)^{q} t^{\prime q}\right) & \text { if } 0<q<\frac{1}{2}\end{cases} \\
& \text { and } \\
& v^{\prime}(T(x)-f(x), t) \leq\left\{\begin{array}{ll}
\sup _{t^{\prime}<t} v\left(x,\left(\frac{2-2^{p}}{3}\right)^{q} t^{\prime q}\right) & \text { if } q>1, \\
\sup _{t^{\prime}<t} v\left(x,\left(\frac{\left(4-2^{p}\right)\left(2-2^{p}\right)}{6}\right)^{q} t^{\prime q}\right) & \text { if } \frac{1}{2}<q<1, \\
\sup _{t^{\prime}<t} v\left(x,\left(\frac{2^{p}-4}{3}\right)^{q} t^{\prime q}\right) & \text { if } 0<q<\frac{1}{2},
\end{array}\right\}
\end{aligned}
$$

for all $x \in X$ and all $t>0$ with $t^{\prime} \in(0, t)$, where $p=1 / q$.

Proof Putting $x=0=y=z$ in (3.1), it follows that

$$
\mu^{\prime}(f(0), t) \geq \mu\left(0,(t / 3)^{q}\right) * \mu\left(0,(t / 3)^{q}\right) * \mu\left(0,(t / 3)^{q}\right)=1
$$

and

$$
v^{\prime}(f(0), t) \leq v\left(0,(t / 3)^{q}\right) \diamond v\left(0,(t / 3)^{q}\right) \diamond v\left(0,(t / 3)^{q}\right)=0
$$

for all $t>0$. Using the definition of IFN-space, we have $f(0)=0$. Now we are ready to prove our theorem for three cases. We consider the cases as $q>1, \frac{1}{2}<q<1$ and $0<q<\frac{1}{2}$. 
Case 1. Let $q>1$. Consider a mapping $J_{n} f: X \rightarrow Y$ to be such that

$$
J_{n} f(x)=\frac{1}{2}\left(4^{-n}\left(f\left(2^{n} x\right)+f\left(-2^{n} x\right)\right)+2^{-n}\left(f\left(2^{n} x\right)-f\left(-2^{n} x\right)\right)\right)
$$

for all $x \in X$. Notice that $J_{0} f(x)=f(x)$ and

$$
\begin{aligned}
J_{j} f(x)-J_{j+1} f(x)= & \frac{D f\left(2^{j} x, 2^{j} x,-2^{j} x\right)}{2 \cdot 4^{j+1}}+\frac{D f\left(-2^{j} x,-2^{j} x, 2^{j} x\right)}{2 \cdot 4^{j+1}} \\
& +\frac{D f\left(2^{j} x, 2^{j} x,-2^{j} x\right)}{2^{j+2}}-\frac{D f\left(-2^{j} x,-2^{j} x, 2^{j} x\right)}{2^{j+2}}
\end{aligned}
$$

for all $x \in X$ and $j \geq 0$. Using the definition of IFN-space and (3.1), this equation implies that if $n+m>m \geq 0$, then

$$
\begin{aligned}
& \mu^{\prime}\left(J_{m} f(x)-J_{n+m} f(x), \sum_{j=m}^{n+m-1} \frac{3}{2}\left(\frac{2^{p}}{2}\right)^{j} t^{p}\right) \\
& =\mu^{\prime}\left(\sum_{j=m}^{n+m-1}\left(J_{j} f(x)-J_{j+1} f(x)\right), \sum_{j=m}^{n+m-1} \frac{3 \cdot 2^{j p}}{2^{j+1}} t^{p}\right) \\
& \geq \prod_{j=m}^{n+m-1} \mu^{\prime}\left(J_{j}\left(f(x)-J_{j+1} f(x)\right), \frac{3 \cdot 2^{j p}}{2^{j+1}}\right) \\
& \geq \prod_{j=m}^{n+m-1}\left\{\mu^{\prime}\left(\frac{\left(2^{j+1}+1\right) D f\left(2^{j} x, 2^{j} x,-2^{j} x\right)}{2 \cdot 4^{j+1}}, \frac{3\left(2^{j+1}+1\right) 2^{j p} t^{p}}{2 \cdot 4^{j+1}}\right)\right. \\
& \left.\quad * \mu^{\prime}\left(\frac{1-\left(2^{j+1}\right) D f\left(-2^{j} x,-2^{j} x, 2^{j} x\right)}{2 \cdot 4^{j+1}}, \frac{3\left(2^{j+1}-1\right) 2^{j p} t^{p}}{2 \cdot 4^{j+1}}\right)\right\} \\
& \geq \prod_{j=m}^{n+m-1} \mu\left(2^{j} x, 2^{j} t\right)=\mu(x, t)
\end{aligned}
$$

and

$$
\begin{aligned}
& v^{\prime}\left(J_{m} f(x)-J_{n+m} f(x), \sum_{j=m}^{n+m-1} \frac{3}{2}\left(\frac{2^{p}}{2}\right)^{j} t^{p}\right) \\
& =v^{\prime}\left(\sum_{j=m}^{n+m-1}\left(J_{j} f(x)-J_{j+1} f(x)\right), \sum_{j=m}^{n+m-1} \frac{3 \cdot 2^{j p}}{2^{j+1}} t^{p}\right) \\
& \leq \coprod_{j=m}^{n+m-1} v^{\prime}\left(J_{j}\left(f(x)-J_{j+1} f(x)\right), \frac{3 \cdot 2^{j p}}{2^{j+1}}\right) \\
& \leq \coprod_{j=m}^{n+m-1}\left\{v^{\prime}\left(\frac{\left(2^{j+1}+1\right) D f\left(2^{j} x, 2^{j} x,-2^{j} x\right)}{2 \cdot 4^{j+1}}, \frac{3\left(2^{j+1}+1\right) 2^{j p} t^{p}}{2 \cdot 4^{j+1}}\right)\right. \\
& \left.\diamond v^{\prime}\left(\frac{1-\left(2^{j+1}\right) D f\left(-2^{j} x,-2^{j} x, 2^{j} x\right)}{2 \cdot 4^{j+1}}, \frac{3\left(2^{j+1}-1\right) 2^{j p} t^{p}}{2 \cdot 4^{j+1}}\right)\right\} \\
& \leq \coprod_{j=m}^{n+m-1} v\left(2^{j} x, 2^{j} t\right)=v(x, t)
\end{aligned}
$$


for all $x \in X$ and $t>0$, where $\prod_{j=1}^{n} a_{j}=a_{1} * a_{2} * \cdots * a_{n}, \bigsqcup_{j=1}^{n} a_{j}=a_{1} \diamond a_{2} \diamond \cdots \diamond a_{n}$. Let $\epsilon>0$ and $\delta>0$ be given. Since $\lim _{t \rightarrow \infty} \mu(x, t)=1$ and $\lim _{t \rightarrow \infty} v(x, t)=0$, there exists $t_{0}>0$ such that $\mu\left(x, t_{0}\right) \geq 1-\epsilon$ and $v\left(x, t_{0}\right) \leq \epsilon$ for all $x \in X$. We observe that for some $\tilde{t}>t_{0}$, the series $\sum_{j=0}^{\infty} \frac{3 \cdot 2^{j p}}{2^{j+1}} \tilde{t}^{p}$ converges for $p=\frac{1}{q}<1$, there exists some $n_{0} \geq 0$ such that $\sum_{j=m}^{n+m-1} \frac{3 \cdot 2^{j p}}{2^{j+1}} \tilde{t}^{p}<\delta$ for each $m \geq n_{0}$ and $n>0$. Using (3.4) and (3.5), we have

$$
\begin{aligned}
\mu^{\prime}\left(J_{m} f(x)-J_{n+m} f(x), \delta\right) & \geq \mu^{\prime}\left(J_{m} f(x)-J_{n+m} f(x), \sum_{j=m}^{n+m-1} \frac{3 \cdot 2^{j p}}{2^{j+1}} \tilde{t}^{p}\right) \\
& \geq \mu(x, \tilde{t}) \geq \mu\left(x, t_{0}\right) \geq 1-\epsilon
\end{aligned}
$$

and

$$
v^{\prime}\left(J_{m} f(x)-J_{n+m} f(x), \delta\right) \leq v^{\prime}\left(J_{m} f(x)-J_{n+m} f(x), \sum_{j=m}^{n+m-1} \frac{3 \cdot 2^{j p}}{2^{j+1}} \tilde{t}^{p}\right) \leq v(x, \tilde{t}) \leq v\left(x, t_{0}\right) \leq \epsilon
$$

for all $x \in X$ and $\delta>0$. Hence $\left\{J_{n} f(x)\right\}$ is a Cauchy sequence in the fuzzy Banach space $\left(Y, \mu^{\prime}, v^{\prime}\right)$. Thus, we define a mapping $T: X \rightarrow Y$ such that $T(x):=\left(\mu^{\prime}, v^{\prime}\right)-\lim _{n \rightarrow \infty} J_{n} f(x)$ for all $x \in X$. Moreover, if we put $m=0$ in (3.4) and (3.5), we get

$$
\left.\begin{array}{l}
\mu^{\prime}\left(f(x)-J_{n} f(x), t\right) \geq \mu\left(x, \frac{t^{q}}{\left(\sum_{j=0}^{n-1} \frac{3 \cdot 2^{j p}}{j^{j+1}}\right)^{q}}\right) \quad \text { and } \\
\nu^{\prime}\left(f(x)-J_{n} f(x), t\right) \leq v\left(x, \frac{t^{q}}{\left(\sum_{j=0}^{n-1} \frac{3 \cdot j^{j p}}{2^{j+1}}\right) q}\right)
\end{array}\right\}
$$

for all $x \in X$ and $t>0$. Now we have to show that $T$ is quadratic additive. Let $x, y, z \in X$. Then

$$
\begin{aligned}
\mu^{\prime}(D T(x, y, z), t) \geq & \mu^{\prime}\left(\left(T-J_{n} f\right)(x+y+z), \frac{t}{28}\right) * \mu^{\prime}\left(\left(T-J_{n} f\right)(x), \frac{t}{28}\right) \\
& * \mu^{\prime}\left(\left(T-J_{n} f\right)(y), \frac{t}{28}\right) * \mu^{\prime}\left(\left(T-J_{n} f\right)(z), \frac{t}{28}\right) \\
& * \mu^{\prime}\left(\left(J_{n} f-T\right)(x+y), \frac{t}{28}\right) * \mu^{\prime}\left(\left(J_{n} f-T\right)(x+z), \frac{t}{28}\right) \\
& * \mu^{\prime}\left(\left(J_{n} f-T\right)(y+z), \frac{t}{28}\right) * \mu^{\prime}\left(D J_{n} f(x, y, z), \frac{3 t}{4}\right)
\end{aligned}
$$

and

$$
\begin{aligned}
v^{\prime}(D T(x, y, z), t) \leq & v^{\prime}\left(\left(T-J_{n} f\right)(x+y+z), \frac{t}{28}\right) \diamond v^{\prime}\left(\left(T-J_{n} f\right)(x), \frac{t}{28}\right) \\
& \diamond v^{\prime}\left(\left(T-J_{n} f\right)(y), \frac{t}{28}\right) \diamond v^{\prime}\left(\left(T-J_{n} f\right)(z), \frac{t}{28}\right) \\
& \diamond v^{\prime}\left(\left(J_{n} f-T\right)(x+y), \frac{t}{28}\right) \diamond v^{\prime}\left(\left(J_{n} f-T\right)(x+z), \frac{t}{28}\right) \\
& \diamond v^{\prime}\left(\left(J_{n} f-T\right)(y+z), \frac{t}{28}\right) \diamond v^{\prime}\left(D J_{n} f(x, y, z), \frac{3 t}{4}\right)
\end{aligned}
$$


for all $t>0$ and $n \in \mathbb{N}$. Taking the limit as $n \rightarrow \infty$ in the inequalities (3.7) and (3.8), we can see that first seven terms on the right-hand side of (3.7) and (3.8) tend to 1 and 0 , respectively, by using the definition of $T$. It is left to find the value of the last term on the right-hand side of (3.7) and (3.8). By using the definition of $J_{n} f(x)$, write

$$
\begin{aligned}
& \mu^{\prime}\left(D J_{n} f(x, y, z), \frac{3 t}{4}\right) \\
& \geq \mu^{\prime}\left(\frac{D f\left(2^{n} x, 2^{n} y, 2^{n} z\right)}{2 \cdot 4^{n}}, \frac{3 t}{16}\right) * \mu^{\prime}\left(\frac{D f\left(-2^{n} x,-2^{n} y,-2^{n} z\right)}{2 \cdot 4^{n}}, \frac{3 t}{16}\right) \\
& \quad * \mu^{\prime}\left(\frac{D f\left(2^{n} x, 2^{n} y, 2^{n} z\right)}{2 \cdot 2^{n}}, \frac{3 t}{16}\right) * \mu^{\prime}\left(\frac{D f\left(-2^{n} x,-2^{n} y,-2^{n} z\right)}{2 \cdot 2^{n}}, \frac{3 t}{16}\right)
\end{aligned}
$$

and, similarly,

$$
\begin{aligned}
& v^{\prime}\left(D J_{n} f(x, y, z), \frac{3 t}{4}\right) \\
& \leq v^{\prime}\left(\frac{D f\left(2^{n} x, 2^{n} y, 2^{n} z\right)}{2 \cdot 4^{n}}, \frac{3 t}{16}\right) \diamond v^{\prime}\left(\frac{D f\left(-2^{n} x,-2^{n} y,-2^{n} z\right)}{2 \cdot 4^{n}}, \frac{3 t}{16}\right) \\
& \diamond v^{\prime}\left(\frac{D f\left(2^{n} x, 2^{n} y, 2^{n} z\right)}{2 \cdot 2^{n}}, \frac{3 t}{16}\right) \diamond v^{\prime}\left(\frac{D f\left(-2^{n} x,-2^{n} y,-2^{n} z\right)}{2 \cdot 2^{n}}, \frac{3 t}{16}\right)
\end{aligned}
$$

for all $x, y, z \in X, t>0$ and $n \in \mathbb{N}$. Also, from (3.1), we have

$$
\begin{aligned}
& \mu^{\prime}\left(\frac{D f\left( \pm 2^{n} x, \pm 2^{n} y, \pm 2^{n} z\right)}{2 \cdot 4^{n}}, \frac{3 t}{16}\right) \\
& \quad=\mu^{\prime}\left(D f\left( \pm 2^{n} x, \pm 2^{n} y, \pm 2^{n} z\right), \frac{3 \cdot 4^{n} t}{8}\right) \\
& \quad \geq \mu\left(2^{n} x,\left(\frac{4^{n} t}{8}\right)^{q}\right) * \mu\left(2^{n} y,\left(\frac{4^{n} t}{8}\right)^{q}\right) * \mu\left(2^{n} z,\left(\frac{4^{n} t}{8}\right)^{q}\right) \\
& \quad \geq \mu\left(x, 2^{(2 q-1) n-3 q} t^{q}\right) * \mu\left(y, 2^{(2 q-1) n-3 q} t^{q}\right) * \mu\left(z, 2^{(2 q-1) n-3 q} t^{q}\right)
\end{aligned}
$$

and

$$
\begin{aligned}
& \mu^{\prime}\left(\frac{D f\left( \pm 2^{n} x, \pm 2^{n} y, \pm 2^{n} z\right)}{2 \cdot 2^{n}}, \frac{3 t}{16}\right) \\
& \quad \geq \mu\left(x, 2^{(2 q-1) n-3 q} t^{q}\right) * \mu\left(y, 2^{(2 q-1) n-3 q} t^{q}\right) * \mu\left(z, 2^{(2 q-1) n-3 q} t^{q}\right)
\end{aligned}
$$

for all $x, y, z \in X, t>0$ and $n \in \mathbb{N}$. Since $q>1$, therefore (3.9) tends to 1 as $n \rightarrow \infty$ with the help of (3.11) and (3.12). Similarly, by proceeding along the same lines as in (3.11) and (3.12), we can show that (3.10) tends to 0 as $n \rightarrow \infty$. Thus, inequalities (3.7) and (3.8) become

$$
\mu^{\prime}(D T(x, y, z), t)=1 \quad \text { and } \quad v^{\prime}(D T(x, y, z), t)=0
$$

for all $x, y, z \in X$ and $t>0$. Accordingly, $D T(x, y, z)=0$ for all $x, y, z \in X$. Now we approximate the difference between $f$ and $T$ in a fuzzy sense. Choose $\epsilon \in(0,1)$ and $0<t^{\prime}<t$. Since 
$T$ is the intuitionistic fuzzy limit of $\left\{J_{n} f(x)\right\}$ such that

$$
\mu^{\prime}\left(T(x)-J_{n} f(x), t-t^{\prime}\right) \geq 1-\epsilon \quad \text { and } \quad \nu^{\prime}\left(T(x)-J_{n} f(x), t-t^{\prime}\right) \leq \epsilon
$$

for all $x \in X, t>0$ and $n \in \mathbb{N}$. From (3.6), we have

$$
\begin{aligned}
\mu^{\prime}(T(x)-f(x), t) & \geq \mu^{\prime}\left(T(x)-J_{n} f(x), t-t^{\prime}\right) * \mu^{\prime}\left(J_{n} f(x)-f(x), t^{\prime}\right) \\
& \geq(1-\epsilon) * \mu\left(x, \frac{t^{\prime q}}{\left(\sum_{j=0}^{n-1} \frac{3 \cdot 2^{j p}}{2^{j+1}}\right)^{q}}\right) \geq(1-\epsilon) * \mu\left(x,\left(\frac{\left(2-2^{p}\right) t^{\prime}}{3}\right)^{q}\right)
\end{aligned}
$$

and

$$
\begin{aligned}
v^{\prime}(T(x)-f(x), t) & \leq v^{\prime}\left(T(x)-J_{n} f(x), t-t^{\prime}\right) \diamond v^{\prime}\left(J_{n} f(x)-f(x), t^{\prime}\right) \\
& \leq(1-\epsilon) \diamond v\left(x,\left(\frac{\left(2-2^{p}\right) t^{\prime}}{3}\right)^{q}\right) .
\end{aligned}
$$

Since $\epsilon \in(0,1)$ is arbitrary, we get the inequality (3.2) in this case.

To prove the uniqueness of $T$, assume that $T^{\prime}$ is another quadratic-additive mapping from $X$ into $Y$, which satisfies the required inequality, i.e., (3.2). Then, by (3.3), for all $x \in X$ and $n \in \mathbb{N}$,

$$
\left.\begin{array}{l}
T(x)-J_{n} T(x)=\sum_{j=0}^{n-1}\left(J_{j} T(x)-J_{j+1} T(x)\right)=0, \\
T^{\prime}(x)-J_{n} T^{\prime}(x)=\sum_{j=0}^{n-1}\left(J_{j} T^{\prime}(x)-J_{j+1} T^{\prime}(x)\right)=0 .
\end{array}\right\}
$$

Therefore

$$
\begin{aligned}
\mu^{\prime}\left(T(x)-T^{\prime}(x), t\right)= & \mu^{\prime}\left(J_{n} T(x)-J_{n} T^{\prime}(x), t\right) \\
\geq & \mu^{\prime}\left(J_{n} T(x)-J_{n} f(x), \frac{t}{2}\right) * \mu^{\prime}\left(J_{n} f(x)-J_{n} T^{\prime}(x), \frac{t}{2}\right) \\
\geq & \mu^{\prime}\left(\frac{(T-f)\left(2^{n} x\right)}{2 \cdot 4^{n}}, \frac{t}{8}\right) * \mu^{\prime}\left(\frac{\left(f-T^{\prime}\right)\left(2^{n} x\right)}{2 \cdot 4^{n}}, \frac{t}{8}\right) \\
& * \mu^{\prime}\left(\frac{(T-f)\left(-2^{n} x\right)}{2 \cdot 4^{n}}, \frac{t}{8}\right) * \mu^{\prime}\left(\frac{\left(f-T^{\prime}\right)\left(-2^{n} x\right)}{2 \cdot 4^{n}}, \frac{t}{8}\right) \\
& * \mu^{\prime}\left(\frac{(T-f)\left(2^{n} x\right)}{2 \cdot 2^{n}}, \frac{t}{8}\right) * \mu^{\prime}\left(\frac{\left(f-T^{\prime}\right)\left(2^{n} x\right)}{2 \cdot 2^{n}}, \frac{t}{8}\right) \\
& * \mu^{\prime}\left(\frac{(T-f)\left(-2^{n} x\right)}{2 \cdot 2^{n}}, \frac{t}{8}\right) * \mu^{\prime}\left(\frac{\left(f-T^{\prime}\right)\left(-2^{n} x\right)}{2 \cdot 2^{n}}, \frac{t}{8}\right) \\
\geq & \sup _{t^{\prime}<t} \mu\left(x, 2^{(q-1) n-2 q}\left(\frac{2-2^{p}}{3}\right)^{q} t^{\prime q}\right)
\end{aligned}
$$

and

$$
\begin{aligned}
v^{\prime}\left(T(x)-T^{\prime}(x), t\right) & =v^{\prime}\left(J_{n} T(x)-J_{n} T^{\prime}(x), t\right) \\
& \leq v^{\prime}\left(J_{n} T(x)-J_{n} f(x), \frac{t}{2}\right) \diamond v^{\prime}\left(J_{n} f(x)-J_{n} T^{\prime}(x), \frac{t}{2}\right)
\end{aligned}
$$




$$
\begin{aligned}
\leq & v^{\prime}\left(\frac{(T-f)\left(2^{n} x\right)}{2 \cdot 4^{n}}, \frac{t}{8}\right) \diamond v^{\prime}\left(\frac{\left(f-T^{\prime}\right)\left(2^{n} x\right)}{2 \cdot 4^{n}}, \frac{t}{8}\right) \\
& \diamond v^{\prime}\left(\frac{(T-f)\left(-2^{n} x\right)}{2 \cdot 4^{n}}, \frac{t}{8}\right) \diamond v^{\prime}\left(\frac{\left(f-T^{\prime}\right)\left(-2^{n} x\right)}{2 \cdot 4^{n}}, \frac{t}{8}\right) \\
& \diamond v^{\prime}\left(\frac{(T-f)\left(2^{n} x\right)}{2 \cdot 2^{n}}, \frac{t}{8}\right) \diamond v^{\prime}\left(\frac{\left(f-T^{\prime}\right)\left(2^{n} x\right)}{2 \cdot 2^{n}}, \frac{t}{8}\right) \\
& \diamond v^{\prime}\left(\frac{(T-f)\left(-2^{n} x\right)}{2 \cdot 2^{n}}, \frac{t}{8}\right) \diamond v^{\prime}\left(\frac{\left(f-T^{\prime}\right)\left(-2^{n} x\right)}{2 \cdot 2^{n}}, \frac{t}{8}\right) \\
\leq & \sup _{t^{\prime}<t} v\left(x, 2^{(q-1) n-2 q}\left(\frac{2-2^{p}}{3}\right)^{q} t^{\prime q}\right)
\end{aligned}
$$

for all $x \in X, t>0$ and $n \in \mathbb{N}$. Since $q=1 / p>1$ and taking limit as $n \rightarrow \infty$ in the last two inequalities, we get $\mu^{\prime}\left(T(x)-T^{\prime}(x), t\right)=1$ and $\nu^{\prime}\left(T(x)-T^{\prime}(x), t\right)=0$ for all $x \in X$ and $t>0$. Hence $T(x)=T^{\prime}(x)$ for all $x \in X$.

Case 2. Let $\frac{1}{2}<q<1$. Consider a mapping $J_{n} f: X \rightarrow Y$ to be such that

$$
J_{n} f(x)=\frac{1}{2}\left(4^{-n}\left(f\left(2^{n} x\right)+f\left(-2^{n} x\right)\right)+2^{n}\left(f\left(\frac{x}{2^{n}}\right)-f\left(-\frac{x}{2^{n}}\right)\right)\right)
$$

for all $x \in X$. Then $J_{0} f(x)=f(x)$ and

$$
\begin{aligned}
J_{j} f(x)-J_{j+1} f(x)= & \frac{D f\left(-2^{j} x,-2^{j} x, 2^{j} x\right)}{2 \cdot 4^{j+1}}+\frac{D f\left(2^{j} x, 2^{j} x,-2^{j} x\right)}{2 \cdot 4^{j+1}} \\
& -2^{j-1}\left(D f\left(\frac{x}{2^{j+1}}, \frac{x}{2^{j+1}}, \frac{-x}{2^{j+1}}\right)-D f\left(\frac{-x}{2^{j+1}}, \frac{-x}{2^{j+1}}, \frac{x}{2^{j+1}}\right)\right)
\end{aligned}
$$

for all $x \in X$ and $j \geq 0$. Thus, for each $n+m>m \geq 0$, we have

$$
\begin{aligned}
& \mu^{\prime}\left(J_{m} f(x)-J_{n+m} f(x), \sum_{j=m}^{n+m-1}\left(\frac{3}{4}\left(\frac{2^{p}}{4}\right)^{j}+\frac{3}{2^{p}}\left(\frac{2}{2^{p}}\right)^{j}\right) t^{p}\right) \\
& \geq \prod_{j=m}^{n+m-1}\left\{\mu^{\prime}\left(\frac{D f\left(2^{j} x, 2^{j} x,-2^{j} x\right)}{2 \cdot 4^{j+1}}, \frac{3 \cdot 2^{j p} t^{p}}{2 \cdot 4^{j+1}}\right) * \mu^{\prime}\left(\frac{D f\left(-2^{j} x,-2^{j} x, 2^{j} x\right)}{2 \cdot 4^{j+1}}, \frac{3 \cdot 2^{j p} t^{p}}{2 \cdot 4^{(j+1)}}\right)\right. \\
& \quad * \mu^{\prime}\left(-2^{j-1} D f\left(\frac{x}{2^{j+1}}, \frac{x}{2^{j+1}}, \frac{-x}{2^{j+1}}\right), \frac{3 \cdot 2^{j-1} t^{p}}{2^{(j+1)^{p}}}\right) \\
& \left.\quad * \mu^{\prime}\left(2^{j-1} D f\left(\frac{-x}{2^{j+1}}, \frac{-x}{2^{j+1}}, \frac{x}{2^{j+1}}\right), \frac{3 \cdot 2^{j-1} t^{p}}{2^{(j+1)^{p}}}\right)\right\} \\
& \geq \prod_{j=m}^{n+m-1}\left\{\mu\left(2^{j} x, 2^{j} t\right) * \mu\left(\frac{x}{2^{j+1}}, \frac{t}{2^{j+1}}\right)\right\}=\mu(x, t) \text { and } \\
& v^{\prime}\left(J_{m} f(x)-J_{n+m} f(x), \sum_{j=m}^{n+m-1}\left(\frac{3}{4}\left(\frac{2^{p}}{4}\right){ }^{j}+\frac{3}{2^{p}}\left(\frac{2}{2^{p}}\right)^{j}\right) t^{p}\right) \\
& \quad \leq \coprod_{j=m}^{n+m-1}\left\{v^{\prime}\left(\frac{D f\left(2^{j} x, 2^{j} x,-2^{j} x\right)}{2 \cdot 4^{j+1}}, \frac{3 \cdot 2^{j p} t^{p}}{2 \cdot 4^{j+1}}\right) \diamond v^{\prime}\left(\frac{D f\left(-2^{j} x,-2^{j} x, 2^{j} x\right)}{2 \cdot 4^{j+1}}, \frac{3 \cdot 2^{j p} t^{p}}{2 \cdot 4^{(j+1)}}\right)\right. \\
& \quad \diamond v^{\prime}\left(-2^{j-1} D f\left(\frac{x}{2^{j+1}}, \frac{x}{2^{j+1}}, \frac{-x}{2^{j+1}}\right), \frac{3 \cdot 2^{j-1} t^{p}}{2^{(j+1)^{p}}}\right)
\end{aligned}
$$




$$
\begin{aligned}
& \left.\diamond v^{\prime}\left(2^{j-1} D f\left(\frac{-x}{2^{j+1}}, \frac{-x}{2^{j+1}}, \frac{x}{2^{j+1}}\right), \frac{3 \cdot 2^{j-1} t^{p}}{2^{(j+1)^{p}}}\right)\right\} \\
\leq & \coprod_{j=m}^{n+m-1}\left\{v\left(2^{j} x, 2^{j} t\right) \diamond v\left(\frac{x}{2^{j+1}}, \frac{t}{2^{j+1}}\right)\right\}=v(x, t),
\end{aligned}
$$

where $\prod$ and $\amalg$ are the same as in Case 1. Proceeding along a similar argument as in Case 1, we see that $\left\{J_{n} f(x)\right\}$ is a Cauchy sequence in $\left(Y, \mu^{\prime}, v^{\prime}\right)$. Thus, we define $T(x):=$ $\left(\mu^{\prime}, v^{\prime}\right)-\lim _{n \rightarrow \infty} J_{n} f(x)$ for all $x \in X$. Putting $m=0$ in the last two inequalities, we get

$$
\left.\begin{array}{l}
\mu^{\prime}\left(f(x)-J_{n} f(x), t\right) \geq \mu\left(x, \frac{t^{p}}{\left(\sum_{j=0}^{n-1}\left(\frac{3}{4}\left(\frac{2^{p}}{4}\right)^{j}+\frac{3}{2^{p}}\left(\frac{2}{2^{p}}\right)^{j}\right)\right)^{q}}\right) \quad \text { and } \\
v^{\prime}\left(f(x)-J_{n} f(x), t\right) \leq v\left(x, \frac{t^{p}}{\left(\sum_{j=0}^{n-1}\left(\frac{3}{4}\left(\frac{2^{p}}{4}\right)^{j}+\frac{3}{2^{p}}\left(\frac{2}{2^{p}}\right)^{j}\right)\right)^{q}}\right)
\end{array}\right\}
$$

for all $x \in X$ and $t>0$. To prove that $t$ is a quadratic-additive function, it is enough to show that the last term on the right-hand side of (3.7) and (3.8) tends to 1 and 0 , respectively, as $n \rightarrow \infty$. Using the definition of $J_{n} f(x)$ and (3.1), we obtain

$$
\begin{aligned}
\mu^{\prime} & \left(D J_{n} f(x, y, z), \frac{3 t}{4}\right) \\
\geq & \mu^{\prime}\left(\frac{D f\left(2^{n} x, 2^{n} y, 2^{n} z\right)}{2 \cdot 4^{n}}, \frac{3 t}{16}\right) * \mu^{\prime}\left(\frac{D f\left(-2^{n} x,-2^{n} y,-2^{n} z\right)}{2 \cdot 4^{n}}, \frac{3 t}{16}\right) \\
& * \mu^{\prime}\left(2^{n-1} D f\left(\frac{x}{2^{n}}, \frac{y}{2^{n}}, \frac{z}{2^{n}}\right), \frac{3 t}{16}\right) * \mu^{\prime}\left(2^{n-1} D f\left(\frac{-x}{2^{n}}, \frac{-y}{2^{n}}, \frac{-z}{2^{n}}\right), \frac{3 t}{16}\right) \\
\geq & \mu\left(x, 2^{(2 q-1) n-3 q} t^{q}\right) * \mu\left(y, 2^{(2 q-1) n-3 q} t^{q}\right) * \mu\left(z, 2^{(2 q-1) n-3 q} t^{q}\right) \\
& * \mu\left(x, 2^{(1-q) n-3 q} t^{q}\right) * \mu\left(y, 2^{(1-q) n-3 q} t^{q}\right) * \mu\left(z, 2^{(1-q) n-3 q} t^{q}\right)
\end{aligned}
$$

and

$$
\begin{aligned}
& v^{\prime}\left(D J_{n} f(x, y, z), \frac{3 t}{4}\right) \\
& \leq v^{\prime}\left(\frac{D f\left(2^{n} x, 2^{n} y, 2^{n} z\right)}{2 \cdot 4^{n}}, \frac{3 t}{16}\right) \diamond v^{\prime}\left(\frac{D f\left(-2^{n} x,-2^{n} y,-2^{n} z\right)}{2 \cdot 4^{n}}, \frac{3 t}{16}\right) \\
& \diamond v^{\prime}\left(2^{n-1} D f\left(\frac{x}{2^{n}}, \frac{y}{2^{n}}, \frac{z}{2^{n}}\right), \frac{3 t}{16}\right) \diamond v^{\prime}\left(2^{n-1} D f\left(\frac{-x}{2^{n}}, \frac{-y}{2^{n}}, \frac{-z}{2^{n}}\right), \frac{3 t}{16}\right) \\
& \leq v\left(x, 2^{(2 q-1) n-3 q} t^{q}\right) \diamond v\left(y, 2^{(2 q-1) n-3 q} t^{q}\right) \diamond v\left(z, 2^{(2 q-1) n-3 q} t^{q}\right) \\
& \diamond v\left(x, 2^{(1-q) n-3 q} t^{q}\right) \diamond v\left(y, 2^{(1-q) n-3 q} t^{q}\right) \diamond v\left(z, 2^{(1-q) n-3 q} t^{q}\right)
\end{aligned}
$$

for each $x, y, z \in X, t>0$ and $n \in \mathbb{N}$. Since $1 / 2<q<1$ and taking the limit as $n \rightarrow \infty$, we see that (3.15) and (3.16) tend to 1 and 0 , respectively. As in Case 1 , we have $D T(x, y, z)=0$ for all $x, y, z \in X$. Using the same argument as in Case 1, we see that (3.2) follows from (3.14). To prove the uniqueness of $T$, assume that $T^{\prime}$ is another quadratic-additive mapping from $X$ into $Y$ satisfying (3.2). Using (3.2) and (3.13), we have

$$
\begin{aligned}
\mu^{\prime}\left(T(x)-T^{\prime}(x), t\right) & =\mu^{\prime}\left(J_{n} T(x)-J_{n} T^{\prime}(x), t\right) \\
& \geq \mu^{\prime}\left(J_{n} T(x)-J_{n} f(x), \frac{t}{2}\right) * \mu^{\prime}\left(J_{n} f(x)-J_{n} T^{\prime}(x), \frac{t}{2}\right)
\end{aligned}
$$




$$
\begin{aligned}
\geq & \mu^{\prime}\left(\frac{(T-f)\left(2^{n} x\right)}{2 \cdot 4^{n}}, \frac{t}{8}\right) * \mu^{\prime}\left(\frac{\left(f-T^{\prime}\right)\left(2^{n} x\right)}{2 \cdot 4^{n}}, \frac{t}{8}\right) \\
& * \mu^{\prime}\left(\frac{(T-f)\left(-2^{n} x\right)}{2 \cdot 4^{n}}, \frac{t}{8}\right) * \mu^{\prime}\left(\frac{\left(f-T^{\prime}\right)\left(-2^{n} x\right)}{2 \cdot 4^{n}}, \frac{t}{8}\right) \\
& * \mu^{\prime}\left(2^{n-1}\left((T-f)\left(\frac{x}{2^{n}}\right)\right), \frac{t}{8}\right) * \mu^{\prime}\left(2^{n-1}\left(\left(f-T^{\prime}\right)\left(\frac{x}{2^{n}}\right)\right), \frac{t}{8}\right) \\
& * \mu^{\prime}\left(2^{n-1}\left((T-f)\left(\frac{-x}{2^{n}}\right)\right), \frac{t}{8}\right) * \mu^{\prime}\left(2^{n-1}\left(\left(f-T^{\prime}\right)\left(\frac{-x}{2^{n}}\right)\right), \frac{t}{8}\right) \\
\geq & \sup _{t^{\prime}<t} \mu\left(x, 2^{(2 q-1) n-2 q}\left(\frac{\left(4-2^{p}\right)\left(2^{p}-2\right)}{6}\right)^{q} t^{\prime q}\right) \\
& * \sup _{t^{\prime}<t} \mu\left(x, 2^{2(1-q) n-2 q}\left(\frac{\left(4-2^{p}\right)\left(2^{p}-2\right)}{6}\right)^{q} t^{\prime q}\right)
\end{aligned}
$$

and

$$
\begin{aligned}
v^{\prime}\left(T(x)-T^{\prime}(x), t\right) \leq & v^{\prime}\left(J_{n} T(x)-J_{n} f(x), \frac{t}{2}\right) \diamond v^{\prime}\left(J_{n} f(x)-J_{n} T^{\prime}(x), \frac{t}{2}\right) \\
\leq & v^{\prime}\left(\frac{(T-f)\left(2^{n} x\right)}{2 \cdot 4^{n}}, \frac{t}{8}\right) \diamond v^{\prime}\left(\frac{\left(f-T^{\prime}\right)\left(2^{n} x\right)}{2 \cdot 4^{n}}, \frac{t}{8}\right) \\
& \diamond v^{\prime}\left(\frac{(T-f)\left(-2^{n} x\right)}{2 \cdot 4^{n}}, \frac{t}{8}\right) \diamond v^{\prime}\left(\frac{\left(f-T^{\prime}\right)\left(-2^{n} x\right)}{2 \cdot 4^{n}}, \frac{t}{8}\right) \\
& \diamond v^{\prime}\left(2^{n-1}\left((T-f)\left(\frac{x}{2^{n}}\right)\right), \frac{t}{8}\right) \diamond v^{\prime}\left(2^{n-1}\left(\left(f-T^{\prime}\right)\left(\frac{x}{2^{n}}\right)\right), \frac{t}{8}\right) \\
& \diamond v^{\prime}\left(2^{n-1}\left((T-f)\left(\frac{-x}{2^{n}}\right)\right), \frac{t}{8}\right) \diamond v^{\prime}\left(2^{n-1}\left(\left(f-T^{\prime}\right)\left(\frac{-x}{2^{n}}\right)\right), \frac{t}{8}\right) \\
\leq & \sup _{t^{\prime}<t} \mu\left(x, 2^{(2 q-1) n-2 q}\left(\frac{\left(4-2^{p}\right)\left(2^{p}-2\right)}{6}\right)^{q} t^{\prime q}\right) \\
& \diamond \sup _{t^{\prime}<t} \mu\left(x, 2^{2(1-q) n-2 q}\left(\frac{\left(4-2^{p}\right)\left(2^{p}-2\right)}{6}\right)^{q} t^{\prime q}\right)
\end{aligned}
$$

for all $x \in X, t>0$ and $n \in \mathbb{N}$. Letting $n \rightarrow \infty$ in (3.17) and (3.18), and using the fact that $\lim _{n \rightarrow \infty} 2^{(2 q-1) n-2 q}=\lim _{n \rightarrow \infty} 2^{(1-q) n-2 q}=\infty$ together with the definition of IFN-space, we get $\mu^{\prime}\left(T(x)-T^{\prime}(x), t\right)=1$ and $\nu^{\prime}\left(T(x)-T^{\prime}(x), t\right)=0$ for all $x \in X$ and $t>0$. Hence $T(x)=$ $T^{\prime}(x)$ for all $x \in X$.

Case 3. Let $0<q<\frac{1}{2}$. Define a mapping $J_{n} f: X \rightarrow Y$ by

$$
J_{n} f(x)=\frac{1}{2}\left(4^{n}\left(f\left(2^{-n} x\right)+f\left(-2^{-n} x\right)\right)+2^{n}\left(f\left(\frac{x}{2^{n}}\right)-f\left(-\frac{x}{2^{n}}\right)\right)\right)
$$

for all $x \in X$. In this case, $J_{0} f(x)=f(x)$ and

$$
\begin{aligned}
J_{j} f(x)-J_{j+1} f(x)= & -\frac{4^{j}}{2}\left(D f\left(\frac{-x}{2^{j+1}}, \frac{-x}{2^{j+1}}, \frac{x}{2^{j+1}}\right)+D f\left(\frac{x}{2^{j+1}}, \frac{x}{2^{j+1}}, \frac{-x}{2^{j+1}}\right)\right) \\
& -2^{j-1}\left(D f\left(\frac{x}{2^{j+1}}, \frac{x}{2^{j+1}}, \frac{-x}{2^{j+1}}\right)-D f\left(\frac{-x}{2^{j+1}}, \frac{-x}{2^{j+1}}, \frac{x}{2^{j+1}}\right)\right)
\end{aligned}
$$


for all $x \in X$ and $j \geq 0$. Thus, for each $n+m>m \geq 0$, we have

$$
\begin{aligned}
\mu^{\prime} & \left(J_{m} f(x)-J_{n+m} f(x) \sum_{j=m}^{n+m-1} \frac{3}{2^{p}}\left(\frac{4}{2^{p}}\right)^{j} t^{p}\right) \\
\geq & \prod_{j=m}^{n+m-1}\left\{\mu^{\prime}\left(-\frac{\left(4^{j}+2^{j}\right) D f\left(\frac{x}{2^{j+1}}, \frac{x}{2^{j+1}}, \frac{-x}{2^{j+1}}\right)}{2}, \frac{3\left(4^{j}+2^{j}\right) t^{p}}{2 \cdot 2^{(j+1)^{p}}}\right)\right. \\
\geq & \left.\prod_{j=m}^{*} \mu^{\prime}\left(-\frac{\left(4^{j}-2^{j}\right) D f\left(\frac{-x}{2^{j+1}}, \frac{-x}{2^{j+1}}, \frac{x}{2^{j+1}}\right)}{2}, \frac{3\left(4^{j}-2^{j}\right) t^{p}}{2 \cdot 2^{(j+1)^{p}}}\right)\right\} \\
v^{\prime} & \left.\left(J_{m} f(x)-J_{n+m} f(x) \sum_{j=m}^{n+m-1} \frac{t}{2^{j+1}}\right)=\mu\left(\frac{4}{2^{p}}\right)^{j} t^{p}\right) \\
\leq & \coprod_{j=m}^{n+m-1}\left\{v^{\prime}\left(-\frac{\left(4^{j}+2^{j}\right) D f\left(\frac{x}{2^{j+1}}, \frac{x}{2^{j+1}}, \frac{-x}{2^{j+1}}\right)}{2}, \frac{3\left(4^{j}+2^{j}\right) t^{p}}{2 \cdot 2^{(j+1)^{p}}}\right)\right. \\
\leq & \coprod_{j=m}^{\diamond} v\left(\frac{x}{2^{j+1}}, \frac{t}{2^{j+1}}\right)=v(x, t) \\
& \left.v^{n+m-1}\left(-\frac{\left(4^{j}-2^{j}\right) D f\left(\frac{-x}{2^{j+1}}, \frac{-x}{2^{j+1}}, \frac{x}{2^{j+1}}\right)}{2}, \frac{3\left(4^{j}-2^{j}\right) t^{p}}{2 \cdot 2^{(j+1)^{p}}}\right)\right\}
\end{aligned}
$$

for all $x \in X$ and $t>0$. Proceeding along a similar argument as in the previous cases, we see that $\left\{J_{n} f(x)\right\}$ is a Cauchy sequence in $\left(Y, \mu^{\prime}, v^{\prime}\right)$. Thus, we define $T(x):=\left(\mu^{\prime}, v^{\prime}\right)-$ $\lim _{n \rightarrow \infty} J_{n} f(x)$ for all $x \in X$. Putting $m=0$ in the last two inequalities, we get

$$
\left.\begin{array}{ll}
\mu^{\prime}\left(f(x)-J_{n} f(x), t\right) \geq \mu\left(x, \frac{t^{q}}{\left(\sum_{j=0}^{n-1} \frac{3}{2^{p}}\left(\frac{4}{2^{p}}\right)^{j}\right)^{q}}\right) & \text { and } \\
v^{\prime}\left(f(x)-J_{n} f(x), t\right) \leq v\left(x, \frac{t^{q}}{\left(\sum_{j=0}^{n-1} \frac{3}{2^{p}}\left(\frac{4}{2^{p}}\right)^{j}\right)^{q}}\right)
\end{array}\right\}
$$

for all $x \in X$ and $t>0$. Write

$$
\begin{aligned}
& \mu^{\prime}\left(D J_{n} f(x, y, z), \frac{3 t}{4}\right) \\
& \geq \mu^{\prime}\left(\frac{4^{n}}{2} D f\left(\frac{x}{2^{n}}, \frac{y}{2^{n}}, \frac{z}{2^{n}}\right), \frac{3 t}{16}\right) \\
& \quad * \mu^{\prime}\left(\frac{4^{n}}{2} D f\left(\frac{-x}{2^{n}}, \frac{-y}{2^{n}}, \frac{-z}{2^{n}}\right), \frac{3 t}{16}\right) \\
& \quad * \mu^{\prime}\left(2^{n-1} D f\left(\frac{x}{2^{n}}, \frac{y}{2^{n}}, \frac{z}{2^{n}}\right), \frac{3 t}{16}\right) \\
& \quad * \mu^{\prime}\left(2^{n-1} D f\left(\frac{-x}{2^{n}}, \frac{-y}{2^{n}}, \frac{-z}{2^{n}}\right), \frac{3 t}{16}\right) \\
& \geq \mu\left(x, 2^{(1-2 q) n-3 q} t^{q}\right) * \mu\left(y, 2^{(1-2 q) n-3 q} t^{q}\right) * \mu\left(z, 2^{(1-2 q) n-3 q} t^{q}\right) \\
& \quad * \mu\left(x, 2^{(1-q) n-3 q} t^{q}\right) * \mu\left(y, 2^{(1-q) n-3 q} t^{q}\right) * \mu\left(z, 2^{(1-q) n-3 q} t^{q}\right)
\end{aligned}
$$


and

$$
\begin{aligned}
& v^{\prime}\left.D J_{n} f(x, y, z), \frac{3 t}{4}\right) \\
& \leq v^{\prime}\left(\frac{4^{n}}{2} D f\left(\frac{x}{2^{n}}, \frac{y}{2^{n}}, \frac{z}{2^{n}}\right), \frac{3 t}{16}\right) \diamond v^{\prime}\left(\frac{4^{n}}{2} D f\left(\frac{-x}{2^{n}}, \frac{-y}{2^{n}}, \frac{-z}{2^{n}}\right), \frac{3 t}{16}\right) \\
& \diamond v^{\prime}\left(2^{n-1} D f\left(\frac{x}{2^{n}}, \frac{y}{2^{n}}, \frac{z}{2^{n}}\right), \frac{3 t}{16}\right) \diamond v^{\prime}\left(2^{n-1} D f\left(\frac{-x}{2^{n}}, \frac{-y}{2^{n}}, \frac{-z}{2^{n}}\right), \frac{3 t}{16}\right) \\
& \leq v\left(x, 2^{(1-2 q) n-3 q} t^{q}\right) \diamond v\left(y, 2^{(1-2 q) n-3 q} t^{q}\right) \diamond v\left(z, 2^{(1-2 q) n-3 q} t^{q}\right) \\
& \diamond v\left(x, 2^{(1-q) n-3 q} t^{q}\right) \diamond v\left(y, 2^{(1-q) n-3 q} t^{q}\right) \diamond v\left(z, 2^{(1-q) n-3 q} t^{q}\right)
\end{aligned}
$$

for all $x, y, z \in X, t>0$ and $n \in \mathbb{N}$. Since $1 / 2<q<1$ and taking the limit as $n \rightarrow \infty$, we see that (3.20) and (3.21) tend to 1 and 0 , respectively. As in the previous cases, we have that $D T(x, y, z)=0$ for all $x, y, z \in X$. By the same argument as in previous cases, we can see that (3.2) follows from (3.19). To prove the uniqueness of $T$, assume that $T^{\prime}$ is another quadratic-additive mapping from $X$ into $Y$ satisfying (3.2). From (3.2) and (3.13), for all $x \in X$ and $t>0$, write

$$
\begin{aligned}
\mu^{\prime}\left(T(x)-T^{\prime}(x), t\right)= & \nu^{\prime}\left(J_{n} T(x)-J_{n} T^{\prime}(x), t\right) \\
\geq & \mu^{\prime}\left(J_{n} T(x)-J_{n} f(x), \frac{t}{2}\right) * \mu^{\prime}\left(J_{n} f(x)-J_{n} T^{\prime}(x), \frac{t}{2}\right) \\
\geq & \mu^{\prime}\left(\frac{4^{n}}{2}\left((T-f)\left(\frac{x}{2^{n}}\right)\right), \frac{t}{8}\right) * \mu\left(\frac{4^{n}}{2}\left(\left(f-T^{\prime}\right)\left(\frac{x}{2^{n}}\right)\right), \frac{t}{8}\right) \\
& * \mu^{\prime}\left(\frac{4^{n}}{2}\left((T-f)\left(-\frac{x}{2^{n}}\right)\right), \frac{t}{8}\right) * \mu^{\prime}\left(\frac{4^{n}}{2}\left(\left(f-T^{\prime}\right)\left(-\frac{x}{2^{n}}\right)\right), \frac{t}{8}\right) \\
& * \mu^{\prime}\left(2^{n-1}\left((T-f)\left(\frac{x}{2^{n}}\right)\right), \frac{t}{8}\right) * \mu^{\prime}\left(2^{n-1}\left(\left(f-T^{\prime}\right)\left(\frac{x}{2^{n}}\right)\right), \frac{t}{8}\right) \\
& * \mu^{\prime}\left(2^{n-1}\left((T-f)\left(\frac{-x}{2^{n}}\right)\right), \frac{t}{8}\right) * \mu^{\prime}\left(2^{n-1}\left(\left(f-T^{\prime}\right)\left(\frac{-x}{2^{n}}\right)\right), \frac{t}{8}\right) \\
\geq & \sup _{t^{\prime}<t} \mu\left(x, 2^{(1-2 q) n-2 q}\left(\frac{2^{p}-4}{3}\right){ }^{q} t^{q}\right)
\end{aligned}
$$

and, similarly,

$$
\begin{aligned}
v^{\prime}\left(T(x)-T^{\prime}(x), t\right) \leq & v^{\prime}\left(\frac{4^{n}}{2}\left((T-f)\left(\frac{x}{2^{n}}\right)\right), \frac{t}{8}\right) \diamond v\left(\frac{4^{n}}{2}\left(\left(f-T^{\prime}\right)\left(\frac{x}{2^{n}}\right)\right), \frac{t}{8}\right) \\
& \diamond v^{\prime}\left(\frac{4^{n}}{2}\left((T-f)\left(-\frac{x}{2^{n}}\right)\right), \frac{t}{8}\right) \diamond v^{\prime}\left(\frac{4^{n}}{2}\left(\left(f-T^{\prime}\right)\left(-\frac{x}{2^{n}}\right)\right), \frac{t}{8}\right) \\
& \diamond v^{\prime}\left(2^{n-1}\left((T-f)\left(\frac{x}{2^{n}}\right)\right), \frac{t}{8}\right) \diamond v^{\prime}\left(2^{n-1}\left(\left(f-T^{\prime}\right)\left(\frac{x}{2^{n}}\right)\right), \frac{t}{8}\right) \\
& \diamond v^{\prime}\left(2^{n-1}\left((T-f)\left(\frac{-x}{2^{n}}\right)\right), \frac{t}{8}\right) \diamond v^{\prime}\left(2^{n-1}\left(\left(f-T^{\prime}\right)\left(\frac{-x}{2^{n}}\right)\right), \frac{t}{8}\right) \\
\leq & \sup _{t^{\prime}<t} v\left(x, 2^{(1-2 q) n-2 q}\left(\frac{2^{p}-4}{3}\right)^{q} t^{q}\right)
\end{aligned}
$$


for $n \in \mathbb{N}$. Letting $n \rightarrow \infty$ in (3.17) and (3.18), and using the fact that $\lim _{n \rightarrow \infty} 2^{(2 q-1) n-2 q}=$ $\lim _{n \rightarrow \infty} 2^{(1-q) n-2 q}=\infty$ together with the definition of IFN-space, we get $\mu^{\prime}(T(x)-$ $\left.T^{\prime}(x), t\right)=1$ and $v^{\prime}\left(T(x)-T^{\prime}(x), t\right)=0$ for all $x \in X$ and $t>0$. Hence $T(x)=T^{\prime}(x)$ for all $x \in X$.

Remark 3.2 Let $(X, \mu, v)$ be an IFN-space and $(X, \mu, v)$ be an intuitionistic fuzzy Banach space $\left(Y, \mu^{\prime}, v^{\prime}\right)$. Let $f: X \rightarrow Y$ be a mapping satisfying (3.1) with a real number $q<0$ and for all $t>0$. If we choose a real number $\alpha$ with $0<3 \alpha<t$, then

$$
\begin{aligned}
& \mu^{\prime}(D f(x, y, z), t) \geq \mu^{\prime}(D f(x, y, z), 3 \alpha) \geq \mu\left(x, \alpha^{q}\right) * \mu\left(y, \alpha^{q}\right) * \mu\left(z, \alpha^{q}\right) \quad \text { and } \\
& v^{\prime}(D f(x, y, z), t) \leq v^{\prime}(D f(x, y, z), 3 \alpha) \leq v\left(x, \alpha^{q}\right) \diamond v\left(y, \alpha^{q}\right) \diamond v\left(z, \alpha^{q}\right)
\end{aligned}
$$

for all $x, y, z \in X, t>0$ and $q<0$. Since $q<0$, we have $\lim _{\alpha \rightarrow 0^{+}} \alpha^{q}=\infty$. This implies that

$$
\begin{aligned}
& \lim _{\alpha \rightarrow 0^{+}} \mu\left(x, \alpha^{q}\right)=1=\lim _{\alpha \rightarrow 0^{+}} \mu\left(y, \alpha^{q}\right)=\lim _{\alpha \rightarrow 0^{+}} \mu\left(z, \alpha^{q}\right) \quad \text { and } \\
& \lim _{\alpha \rightarrow 0^{+}} v\left(x, \alpha^{q}\right)=0=\lim _{\alpha \rightarrow 0^{+}} v\left(y, \alpha^{q}\right)=\lim _{\alpha \rightarrow 0^{+}} v\left(z, \alpha^{q}\right) .
\end{aligned}
$$

Thus, we have $\mu^{\prime}(D f(x, y, z), t)=1$ and $\nu^{\prime}(D f(x, y, z), t)=0$ for all $x, y, z \in X$ and $t>0$. Hence $D f(x, y, z)=0$ for all $x, y, z \in X$. In other words, if $f$ is an intuitionistic fuzzy $q$-almost quadratic-additive mapping for the case $q<0$, then $f$ is itself a quadratic-additive mapping.

Corollary 3.3 Suppose that $f$ is an even mapping satisfying the conditions of Theorem 3.1. Then there exists a unique quadratic mapping $T: X \rightarrow Y$ such that

$$
\left.\begin{array}{l}
\mu^{\prime}(T(x)-f(x), t) \geq \sup _{t^{\prime}<t} \mu\left(x,\left(\frac{\left|4-2^{p}\right| t^{\prime}}{3}\right)^{q}\right) \quad \text { and } \\
v^{\prime}(T(x)-f(x), t) \leq \sup _{t^{\prime}<t} v\left(x,\left(\frac{\left|4-2^{p}\right| t^{\prime}}{3}\right)^{q}\right)
\end{array}\right\}
$$

for all $x \in X$ and $t>0$, where $p=1 / q$.

Proof Since $f$ is an even mapping, we get

$$
J_{n} f(x)= \begin{cases}\frac{f\left(2^{n} x\right)+f\left(-2^{n} x\right)}{2 \cdot 4^{n}} & \text { if } q>\frac{1}{2}, \\ \frac{1}{2}\left(4^{n}\left(f\left(2^{-n} x\right)+f\left(-2^{-n} x\right)\right)\right) & \text { if } 0<q<\frac{1}{2},\end{cases}
$$

for all $x \in X$, where $J_{n} f$ is defined as in Theorem 3.1. In this case, $J_{0} f(x)=f(x)$. For all $x \in X$ and $j \in \mathbb{N} \cup\{0\}$, we have

$$
J_{j} f(x)-J_{j+1} f(x)= \begin{cases}\frac{D f\left(2^{j} x, 2^{j} x,-2^{j} x\right)}{2 \cdot 4^{j+1}}+\frac{D f\left(-2 j_{x,-2} j_{x, 2} j_{x}\right.}{2 \cdot 4^{j+1}} & \text { if } q>\frac{1}{2}, \\ -\frac{4^{j}}{2}\left(D f\left(\frac{-x}{2^{j+1}}, \frac{-x}{2^{j+1}}, \frac{x}{2^{j+1}}\right)+D f\left(\frac{x}{2^{j+1}}, \frac{x}{2^{j+1}}, \frac{-x}{2^{j+1}}\right)\right) & \text { if } 0<q<\frac{1}{2} .\end{cases}
$$

Proceeding along the same lines as in Theorem 3.1, we obtain that $T$ is a quadraticadditive function satisfying (3.22). Notice that $T(x):=\left(\mu^{\prime}, v^{\prime}\right)-\lim _{n \rightarrow \infty} J_{n} f(x), T$ is even and $D T(x, y, z)=0$ for all $x, y, z \in X$. Hence, we get

$$
T(x+y)+T(x-y)-2 T(x)-2 T(y)=-D T(x, y,-x)=0
$$

for all $x, y \in X$. It follows that $T$ is a quadratic mapping. 
Corollary 3.4 Suppose that $f$ is an even mapping satisfying the conditions of Theorem 3.1. Then there exists a unique additive mapping $T: X \rightarrow Y$ such that

$$
\left.\begin{array}{ll}
\mu^{\prime}(T(x)-f(x), t) \geq \sup _{t^{\prime}<t} \mu\left(x,\left(\frac{\left|2-2^{p}\right| t^{\prime}}{3}\right)^{q}\right) & \text { and } \\
v^{\prime}(T(x)-f(x), t) \leq \sup _{t^{\prime}<t} v\left(x,\left(\frac{\left|2-2^{p}\right| t^{\prime}}{3}\right)^{q}\right)
\end{array}\right\}
$$

for all $x \in X$ and $t>0$, where $p=1 / q$.

Proof Since $f$ is an odd mapping, we get

$$
J_{n} f(x)= \begin{cases}\frac{f\left(2^{n} x\right)+f\left(-2^{n} x\right)}{2^{n+1}} & \text { if } q>1, \\ 2^{n-1}\left(f\left(2^{-n} x\right)+f\left(-2^{-n} x\right)\right) & \text { if } 0<q<1\end{cases}
$$

for all $x \in X$, where $J_{n} f$ is defined as in Theorem 3.1. Here $J_{0} f(x)=f(x)$. For all $x \in X$ and $j \in \mathbb{N} \cup\{0\}$, we have

$$
J_{j} f(x)-J_{j+1} f(x)= \begin{cases}\frac{D f\left(2^{j} x, 2^{j} x,-2^{j} x\right)}{2^{j+2}}-\frac{D f\left(-2^{j} x,-2^{j} x, 2^{j} x\right)}{2^{2^{+2}}} & \text { if } q>1, \\ -2^{j-1}\left(D f\left(\frac{x}{2^{j+1}}, \frac{x}{2^{j+1}}, \frac{-x}{2^{j+1}}\right)-D f\left(\frac{-x}{2^{j+1}}, \frac{-x}{2^{j+1}}, \frac{x}{2^{j+1}}\right)\right) & \text { if } 0<q<1 .\end{cases}
$$

Proceeding along the same lines as in Theorem 3.1, we obtain that $T$ is a quadratic-additive function satisfying (3.23). Here $T(x):=\left(\mu^{\prime}, v^{\prime}\right)-\lim _{n \rightarrow \infty} J_{n} f(x), T$ is odd and $D T(x, y, z)=0$ for all $x, y, z \in X$. Hence, we obtain

$$
T(x+y)-T(x)-T(y)=D f\left(\frac{x-y}{2}, \frac{x+y}{2}, \frac{-x+y}{2}\right)=0
$$

for all $x, y \in X$. It follows that $T$ is an additive mapping.

\section{Competing interests}

The authors declare that they have no competing interests.

\section{Authors' contributions}

The authors contributed equally and significantly in writing this paper. Both the authors read and approved the final manuscript.

\section{Acknowledgements}

This project was funded by the Deanship of Scientific Research (DSR), King Abdulaziz University, Jeddah, under grant no. (405/130/1433). The authors, therefore, acknowledge with thanks DSR technical and financial support.

Received: 13 March 2013 Accepted: 15 June 2013 Published: 8 July 2013

\section{References}

1. Ulam, SM: A Collection of the Mathematical Problems. Interscience, New York (1960)

2. Hyers, DH: On the stability of the linear functional equation. Proc. Natl. Acad. Sci. USA 27, $222-224$ (1941)

3. Aoki, T: On the stability of the linear transformation in Banach spaces. J. Math. Soc. Jpn. 2, $64-66$ (1950)

4. Rassias, TM: On the stability of the linear mapping in Banach spaces. Proc. Am. Math. Soc. 72, 297-300 (1978)

5. Rassias, TM: On the stability of functional equations and a problem of Ulam. Acta Appl. Math. 62, 123-130 (2000)

6. Agarwal, RP, Xu, B, Zhang, W: Stability of functional equations in single variable. J. Math. Anal. Appl. 288, 852-869 (2003)

7. Gajda, Z: On stability of additive mappings. Int. J. Math. Math. Sci. 14, 431-434 (1991)

8. Găvruta, P: A generalization of the Hyers-Ulam-Rassias stability of approximately additive mappings. J. Math. Anal. Appl. 184, 431-436 (1994)

9. Hyers, DH, Isac, G, Rassias, TM: Stability of Functional Equations in Several Variables. Birkhäuser, Basel (1998)

10. Isac, G, Rassias, TM: On the Hyers-Ulam stability of $\psi$-additive mappings. J. Approx. Theory 72, 131-137 (1993) 
11. Najati, A, Park, C: On the stability of an $n$-dimensional functional equation originating from quadratic forms. Taiwan. J. Math. 12, 1609-1624 (2008)

12. Lu, G, Park, C: Additive functional inequalities in Banach spaces. J. Inequal. Appl. 2012, 294 (2012)

13. Rassias, JM, Kim, H-M: Generalized Hyers-Ulam stability for general additive functional equations in quasi- $\beta$-normed spaces. J. Math. Anal. Appl. 356, 302-309 (2009)

14. Rassias, JM: Solution of a problem of Ulam. J. Approx. Theory 57, 268-273 (1989)

15. Rassias, JM: On the Ulam stability of mixed type mappings on restricted domains. J. Math. Anal. Appl. 276, 747-762 (2002)

16. Dadipour, F, Moslehian, MS, Rassias, JM, Takahasi, S-E: Characterization of a generalized triangle inequality in normed spaces. Nonlinear Anal. 75, 735-741 (2012)

17. Eskandani, GZ, Rassias, JM, Gavruta, P: Generalized Hyers-Ulam stability for a general cubic functional equation in quasi- $\beta$-normed spaces. Asian-Eur. J. Math. 4, 413-425 (2011)

18. Faziev, V, Sahoo, PK: On the stability of Jensen's functional equation on groups. Proc. Indian Acad. Sci. Math. Sci. 117 31-48 (2007)

19. Gordji, ME, Khodaei, H, Rassias, JM: Fixed point methods for the stability of general quadratic functional equation. Fixed Point Theory 12, 71-82 (2011)

20. Jun, KW, Kim, HM: The generalized Hyers-Ulam-Rassias stability of a cubic functional equation. J. Math. Anal. Appl. $274,867-878$ (2002)

21. Jung, SM: Hyers-Ulam-Rassias Stability of Functional Equations in Mathematical Analysis. Hadronic Press, Palm Harbor (2001)

22. Ravi, K, Arunkumar, M, Rassias, JM: Ulam stability for the orthogonally general Euler-Lagrange type functional equation. Int. J. Math. Stat. 3(A08), 36-46 (2008)

23. Saadati, R, Park, C: Non-Archimedean $\mathcal{L}$-fuzzy normed spaces and stability of functional equations. Comput. Math. Appl. 60, 2488-2496 (2010)

24. Xu, TZ, Rassias, MJ, Xu, WX, Rassias, JM: A fixed point approach to the intuitionistic fuzzy stability of quintic and sextic functional equations. Iranian J. Fuzzy Sys. 9, 21-40 (2012)

25. Atanassov, K: Intuitionistic fuzzy sets, VIII ITKR's Session, Sofia, June 1983 (Deposed in Central Science-Technical Library of Bulg. Academy of Science, 1697/84) (in Bulgarian)

26. Saadati, R, Park, JH: On the intuitionistic fuzzy topological spaces. Chaos Solitons Fractals 27, 331-344 (2006)

27. Bag, T, Samanta, SK: Finite dimensional fuzzy normed linear spaces. J. Fuzzy Math. 11(3), 687-705 (2003)

28. Mohiuddine, SA, Danish Lohani, QM: On generalized statistical convergence in intuitionistic fuzzy normed space. Chaos Solitons Fractals 42, 1731-1737 (2009)

29. Mursaleen, M, Karakaya, V, Mohiuddine, SA: Schauder basis, separability, and approximation property in intuitionistic fuzzy normed space. Abstr. Appl. Anal. 2010, Art. ID 131868 (2010)

30. Mursaleen, M, Mohiuddine, SA: Statistical convergence of double sequences in intuitionistic fuzzy normed spaces. Chaos Solitons Fractals 41, 2414-2421 (2009)

31. Mursaleen, M, Mohiuddine, SA: On lacunary statistical convergence with respect to the intuitionistic fuzzy normed space. J. Comput. Appl. Math. 233(2), 142-149 (2009)

32. Mursaleen, M, Mohiuddine, SA: Nonlinear operators between intuitionistic fuzzy normed spaces and Fréchet differentiation. Chaos Solitons Fractals 42, 1010-1015 (2009)

33. Mursaleen, M, Mohiuddine, SA, Edely, OHH: On the ideal convergence of double sequences in intuitionistic fuzzy normed spaces. Comput. Math. Appl. 59, 603-611 (2010)

34. Yilmaz, Y: On some basic properties of differentiation in intuitionistic fuzzy normed spaces. Math. Comput. Model. 52, 448-458 (2010)

35. Jin, SS, Lee, Y-H: Fuzzy stability of a mixed type functional equation. J. Inequal. Appl. 2011, 70 (2011)

36. Mohiuddine, SA, Alotaibi, A: Fuzzy stability of a cubic functional equation via fixed point technique. Adv. Differ. Equ. 2012, 48 (2012)

37. Mohiuddine, SA, Alotaibi, A, Obaid, M: Stability of various functional equations in non-Archimedean intuitionistic fuzzy normed spaces. Discrete Dyn. Nat. Soc. 2012, Article ID 234727 (2012)

38. Mohiuddine, SA, Alghamdi, MA: Stability of functional equation obtained through a fixed-point alternative in intuitionistic fuzzy normed spaces. Adv. Differ. Equ. 2012, 141 (2012)

39. Mohiuddine, SA, Sevli, H: Stability of pexiderized quadratic functional equation in intuitionistic fuzzy normed space. J. Comput. Appl. Math. 235, 2137-2146 (2011)

40. Mohiuddine, SA, Cancan, M, Şevli, H: Intuitionistic fuzzy stability of a Jensen functional equation via fixed point technique. Math. Comput. Model. 54, 2403-2409 (2011)

41. Mohiuddine, SA: Stability of Jensen functional equation in intuitionistic fuzzy normed space. Chaos Solitons Fractals 42, 2989-2996 (2009)

42. Mursaleen, M, Mohiuddine, SA: On stability of a cubic functional equation in intuitionistic fuzzy normed spaces. Chaos Solitons Fractals 42, 2997-3005 (2009)

43. Wang, Z, Rassias, TM: Intuitionistic fuzzy stability of functional equations associated with inner product spaces. Abstr. Appl. Anal. 2011, Article ID 456182 (2011)

44. Xu, TZ, Rassias, JM, Xu, WX: Intuitionistic fuzzy stability of a general mixed additive-cubic equation. J. Math. Phys. 51, $063519(2010)$

45. Xu, TZ, Rassias, JM, Xu, WX: Stability of a general mixed additive-cubic functional equation in non-Archimedean fuzzy normed spaces. J. Math. Phys. 51, 093508 (2010)

46. $\mathrm{Xu}, \mathrm{TZ}$, Rassias, JM: Stability of general multi-Euler-Lagrange quadratic functional equations in non-Archimedean fuzzy normed spaces. Adv. Differ. Equ. 2012, 119 (2012)

47. Alotaibi, A, Mohiuddine, SA: On the stability of a cubic functional equation in random 2-normed spaces. Adv. Differ. Equ. 2012, 39 (2012)

48. Goleț, I: On probabilistic 2-normed spaces. Novi Sad J. Math. 35(1), 95-102 (2005)

49. Mohiuddine, SA, Aiyub, M: Lacunary statistical convergence in random 2-normed spaces. Appl. Math. Inform. Sci. 6(3), 581-585 (2012) 
50. Mursaleen, M: On statistical convergence in random 2-normed spaces. Acta Sci. Math. 76, 101-109 (2010)

51. Mohiuddine, SA, Alotaibi, A, Alsulami, SM: Ideal convergence of double sequences in random 2-normed spaces. Adv. Differ. Equ. 2012, 149 (2012)

52. Jung, S-M: On the Hyers-Ulam stability of the functional equations that have the quadratic property. J. Math. Anal. Appl. 222, 126-137 (1998)

53. Kannappan, P: Quadratic functional equation and inner product spaces. Results Math. 27, 368-372 (1995)

doi:10.1186/1687-1847-2013-203

Cite this article as: Al-Fhaid and Mohiuddine: On the Ulam stability of mixed type QA mappings in IFN-spaces.

Advances in Difference Equations 2013 2013:203.

Submit your manuscript to a SpringerOpen ${ }^{\odot}$ journal and benefit from:

- Convenient online submission

Rigorous peer review

- Immediate publication on acceptance

- Open access: articles freely available online

- High visibility within the field

- Retaining the copyright to your article

Submit your next manuscript at $>$ springeropen.com 